Invited talk at $13^{\text {th }}$ International Conference on Ion Beam Analysis (IBA-13),

Lisbon, 27.7.-1.8.97

session G: "Novel ion beam techniques"

Nucl. Instr. Meth. B 136-138 (1998) 744-750.

\title{
Electron emission channeling with position-sensitive detectors
}

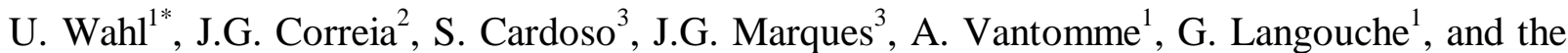
ISOLDE collaboration ${ }^{2}$

1) Instituut voor Kern- en Stralingsfysica, University of Leuven, Celestijnenlaan 200 D,

B-3001 Leuven, Belgium

2) CERN-PPE, CH-1211 Genève 23, Switzerland

3) CFNUL, Av. Prof. Gama Pinto 2, P-1699 Lisboa, Portugal

\begin{abstract}
Electron emission channeling allows direct lattice location studies of low doses of radioactive atoms implanted in single crystals. For that purpose the anisotropic emission yield of conversion electrons from the crystal surface is measured, most conveniently by use of position-sensitive detectors. We discuss characteristic features of this method, including quantitative data analysis procedures, which are achieved by fitting simulated two-dimensional emission distributions for different lattice sites to the experimental patterns. The capabilities of this approach are illustrated by the case of rare earth atoms $(\mathrm{Er}, \mathrm{Tm}, \mathrm{Yb})$ in $\mathrm{Si}$, where we were able to do lattice location experiments down to implanted doses which are 150 times lower compared to previous RBS studies.
\end{abstract}

PACS codes: 61.72.-y, 61.72.Tt, 61.85.+p

Keywords: emission channeling, lattice location, $\mathrm{Si}$, rare earths, ion implantation

* Corresponding author, e-mail: ulrich.wahl@fys.kuleuven.ac.be

phone: ++32-16-327617

fax: $\quad++32-16-327985$

\section{Introduction}

Ion beam channeling is a well-established method to perform direct lattice location studies of foreign atoms incorporated in single crystals, which has found widespread applications during the last 30 years [1]. Typically, lattice location experiments in semiconductors by the Rutherford backscattering (RBS) channeling technique require impurity doses above $10^{14}-10^{15} \mathrm{~cm}^{-2}$ [2,3]. If the impurities are introduced by today's most common doping technique, ion implantation, a heavy-ion dose of $10^{15} \mathrm{~cm}^{-2}$ comes close to amorphizing most semiconductor crystals at room temperature, so that for instance lattice location studies of the as-implanted state are difficult to perform. Light atoms in a heavy matrix suffer from comparable or even more severe limitations since their detection relies on elastic recoil or nuclear reaction analysis.

In many cases, emission channeling and blocking from low doses of radioactive atoms offers an alternative. This method is based on the fact that charged particles from nuclear decay $\left(\alpha, \beta^{+}, \beta\right.$, conversion electrons) experience channeling and blocking effects along major 
crystallographic axes and planes, resulting in an anisotropic emission yield from the crystal surface. It is worthwhile mentioning that this lattice location technique is almost as old as ion beam channeling, and first experiments date back to the mid 1960s [4,5]. The most simple experimental approach to emission channeling is to rotate the sample in front of a collimated particle detector by means of a goniometer, and measure the angular-dependent count rate step by step. A variety of lattice location experiments in metals and semiconductors have been carried out using this technique during the last 15 years [6,7]. However, emission channeling is more conveniently measured by use of position-sensitive detectors (PSDs) with large solid angles, which increases the detection efficiency by about two orders of magnitude. Additional advantages are that there is no need for high-precision computer-controlled goniometers, and that it is no longer required to normalize the counting time per angular position to the sample activity. In the case of $\mathrm{MeV}$ alpha particles, suitable PSD systems were developed already in the 1970s, and they are commercially available from several suppliers. However, alpha emitting isotopes are mainly found, with a few exceptions, at masses above $\approx 150$. On the other hand, beta and conversion electron emitters exist for almost all elements of the periodic system. Position-sensitive detection of these particles represents a greater challenge, though.

\section{General considerations on PSDs for electron emission channeling}

Conversion electrons are emitted from excited nuclear states and have discrete energies, typically of the order of $30-300 \mathrm{keV}$. In order to discriminate contributions from different isotopes or nuclear states and to subtract the background due to backscattered electrons, an energy resolution better than $10 \mathrm{keV}$ is highly desirable. For $\beta^{-}$and $\beta^{+}$particles, which have continuous spectra with end point energies in the range of several hundred $\mathrm{keV}$ to several $\mathrm{MeV}$, a worse energy resolution can be tolerated.

An important characteristic in channeling experiments is the relative angular resolution $\Delta \theta / \theta$. Using a PSD it is limited by both the position resolution $\sigma_{d}$ of the detector and the resolution $\sigma_{\mathrm{b}}$ due to the size of the radioactive spot on the sample. Approximately,

$\Delta \theta / \theta \approx \Delta \theta d / s \approx\left(\sigma_{\mathrm{d}}^{2}+\sigma_{\mathrm{b}}^{2}\right)^{1 / 2} / s$,

where $s$ is either the $x$ - or $y$-size of the detector and $d$ the distance from the sample. A natural limit to the position resolution of the detector is the lateral straggling of the electrons in the detector itself. Its magnitude is comparable to the thickness required to completely stop all electrons, which in $\mathrm{Si}$ is around $7 \mu \mathrm{m}$ at $30 \mathrm{keV}, 350 \mu \mathrm{m}$ at $300 \mathrm{keV}$ and $2 \mathrm{~mm}$ at $1 \mathrm{MeV}$. On the other hand, beam spots much smaller than $1 \mathrm{~mm}$ are difficult to achieve if samples are produced by ion implantation of radioactive atoms. If we require a relative angular resolution better than, say, $10 \%$, it is easily derived from the above equation that we need detectors with at least $\mathrm{cm}$ dimensions and $\mathrm{mm}$ position resolutions.

Si detectors working with the principle of resistive charge division (see, e.g., Ref. [8]) have proven very useful as PSDs for alpha emission channeling [9]. Typically, such devices are $1 \times 1 \mathrm{~cm}^{2}$ to $3 \times 3 \mathrm{~cm}^{2}$ in size and $300-1000 \mu \mathrm{m}$ thick, and energy resolutions down to $16 \mathrm{keV}$ and position resolutions of $70 \mu \mathrm{m}$ for $5.8 \mathrm{MeV}$ alpha particles have been reported [10]. Their relative position resolution $\sigma_{\mathrm{d}} / \mathrm{s}$, however, is approximately equal to their relative energy resolution $\Delta \mathrm{E} / \mathrm{E}[8]$, while the absolute energy resolution $\Delta \mathrm{E}$ is dominated by the noise introduced by the resistance of the charge dividing layer itself and almost independent of particle energy. For energies below $300 \mathrm{keV}$, resistive charge PSDs, therefore, can hardly reach position resolutions better than 5\%, even if operated at their very best noise limit. Hence they are not suited for typical conversion electron experiments, but might be useful for medium energy $\beta^{\text {o }}$ or $\beta^{+}$emitters. 
While Si charged coupled devices (CCDs) for nuclear applications [11] are excellently adapted for position-sensitive detection of conversion electrons, existing prototypes are still rather expensive. Note that multi-channel plates, which are frequently applied as PSDs for keV particles in low energy ion beam analysis, are not suited due to lack of energy resolution.

\section{Si pad detectors}

A promising approach, however, are segmented Si detectors with discrete readout. Such devices are made by integrating an array of separate detector cells ("pads", pixels or strips) on a single Si chip and individually contacting them on the surface by a pattern of conducting and insulating layers. Since the signal to noise of individual cells improves with shrinking cell dimensions due to the decrease in capacitance and leakage current, this allows to downscale energy and position resolution to a large extent. While such detectors are in principle not very difficult to manufacture, their widespread use has been hampered by the high costs of the extensive electronics needed to read out the large number of segments. However, due to the ongoing progress in microelectronics, integrated multichannel preamplifier circuits are meanwhile offered at much lower prices, so that this approach has become feasible at a moderate cost.

In the following we will briefly introduce the PSDs we have used successfully for detection of 40-250 keV conversion electrons; a more detailed description is to be published elsewhere [12]. These systems were developed at CERN in the context of high-energy physics collider experiments [13], and consist of $30 \times 30 \mathrm{~mm}^{2}$ Si detectors of $0.5 \mathrm{~mm}$ or $1 \mathrm{~mm}$ thickness, which are segmented on one side into $22 \times 22$ pads, each of $1.3 \times 1.3 \mathrm{~mm}^{2}$. The 484 pads are coupled to 4 VLSI preamplifier chips of 128 channels each, which are read out by a digital signal processor (DSP) via a $1 \mathrm{MHz}$ sampling analog to digital converter (ADC). Multiplexed read out of all pads is triggered if the signal on the detector backplane, which is common to all pads, exceeds an externally set lower level threshold. This serial readout procedure limits the counting speed of the device to $400 \mathrm{~Hz}$, which is sufficient, however, for typical experiments with long-lived radioactive isotopes above a half life of several hours.

\section{Experimental results and their quantitative analysis}

A series of first experiments was done using the decay chains ${ }^{169} \mathrm{Yb}^{\left(\mathrm{t}_{1 / 2}=32 \mathrm{~d}\right) \rightarrow{ }^{169} \mathrm{Tm}} \mathrm{Tm}^{*}(0.66$ $\mu \mathrm{s})$ and ${ }^{167} \mathrm{Tm}(9.25 \mathrm{~d}) \rightarrow{ }^{167 \mathrm{~m}} \operatorname{Er}(2.28 \mathrm{~s})$. Rare earths were chosen because they are of considerable interest as optical dopants in semiconductors [14]. Furthermore, in the case of $\mathrm{Yb} / \mathrm{Tm}$ we can compare our lattice location results to previous RBS [2,3] and emission channeling [4] studies in Si. Samples were produced at CERN's on-line isotope separator ISOLDE [15] by implanting Si single crystals with $60 \mathrm{keV}$ ions using a $1 \mathrm{~mm}$ beam spot. The angular-dependent emission yield of conversion electrons was measured by the pad detector at a distance of $285 \mathrm{~mm}$ from the sample. From the energy spectrum of the ${ }^{169} \mathrm{Yb}$ decay (Fig. 1) the energy resolution of the detector is found to be $3.2 \mathrm{keV} \mathrm{FWHM}$ for X-rays, and 5-6 keV for electrons. The somewhat worse energy resolution for electrons is due to the energy loss and straggling in the relatively thick detector entrance window $(>2 \mu \mathrm{m})$, which is also responsible for a shift in electron energy of approximately $8 \mathrm{keV}$.

Channeling patterns from ${ }^{169} \mathrm{Yb} /{ }^{169} \mathrm{Tm} *$ were extracted for the part of the spectrum above $98 \mathrm{keV}$. This energy window includes all conversion electrons emitted in the decay of the excited state ${ }^{169} \mathrm{Tm}^{*}(0.66 \mu \mathrm{s})$, which is populated as a consequence of the electron capture (EC) decay of ${ }^{169} \mathrm{Yb}$, but avoids lower energies, where the detector is exceedingly sensitive to X-rays emitted by the sample. To correct for backscattered electrons, a trapezoidal background was subtracted from each conversion electron line. Figs. 2 a), b) and c) show the channeling patterns from a single crystal of $n$-Si:P (1-10 $\Omega \mathrm{cm}, \mathrm{CZ},\langle 100>$ surface) implanted with a dose of $1.2 \times 10^{14} \mathrm{~cm}^{-2}$, followed by annealing at $600^{\circ} \mathrm{C}$ for $10 \mathrm{~min}$. Clearly visible are prominent 


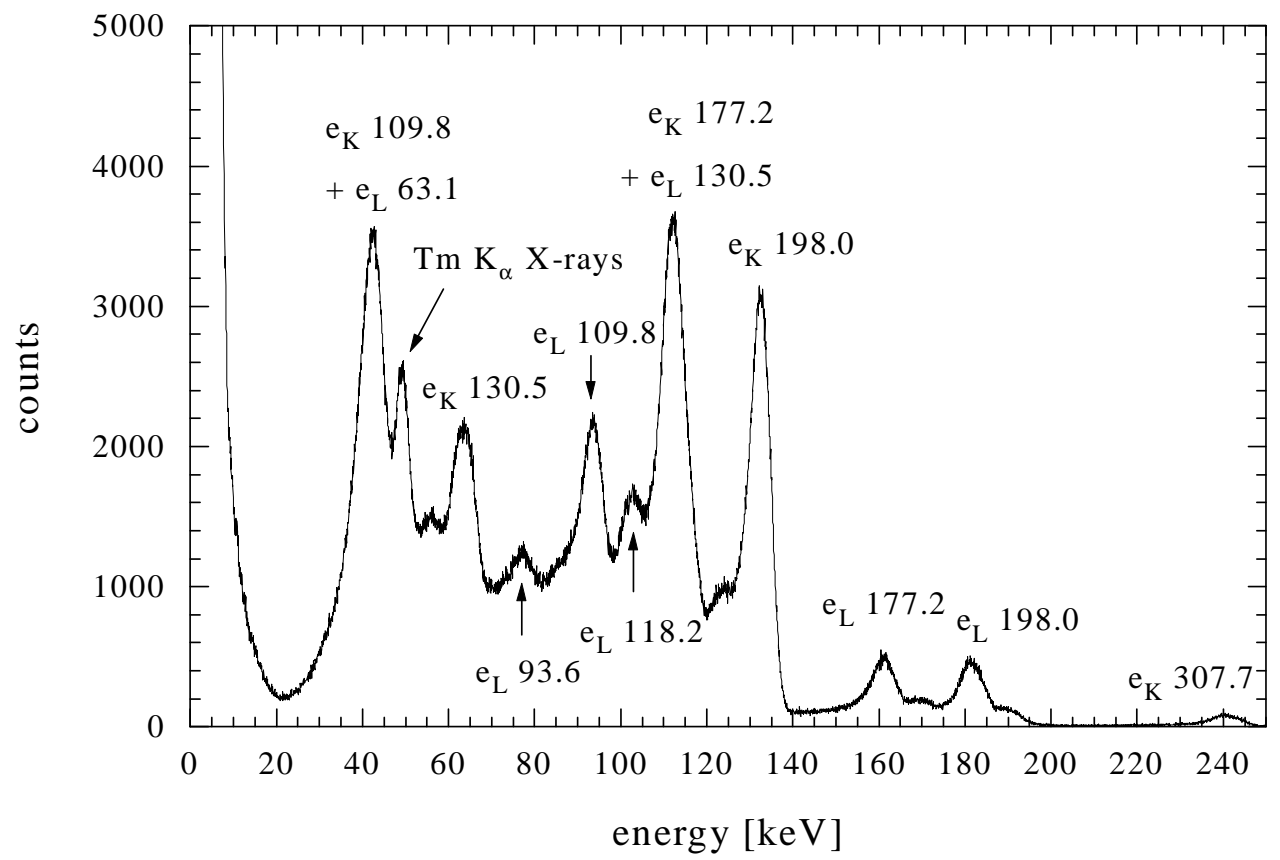

Fig. 1

Fig. 1. Energy spectrum of ${ }^{169} \mathrm{Yb}$, recorded with the $1 \mathrm{~mm}$ thick Si pad detector. Conversion electrons from ${ }^{169} \mathrm{Tm}$ are labeled by the electron shells from which they originate and by the corresponding gamma transitions in $\mathrm{keV}$.

channeling effects along axial <100> and <111> and planar $\{110\}$ directions, and less pronounced channeling effects along $\{100\}$ and $\{211\}$. On the contrary, the axial $\langle 110\rangle$ and planar $\{111\}$ and $\{311\}$ directions all show yields close to unity or below. The combination of these patterns is characteristic for sites close to the tetrahedral interstitial (T) position. While $\mathrm{T}$ sites are perfectly aligned with $\langle 100\rangle,\langle 111\rangle,\{100\},\{110\}$ and $\{211\}$ lattice directions, leading to channeling of electrons, they are interstitial with respect to $\langle 110\rangle$ atomic axes and $\{111\}$ and $\{311\}$ atomic planes, causing yield minima along these directions. Note that due to the negative charge of the electrons this is essentially the opposite behaviour compared to ion beam or alpha emission channeling [9].

In order to identify the lattice site occupation more precisely, we fit the experimental patterns with theoretical emission yields from various lattice sites, which we calculate using the "many beam" approximation of the dynamical theory of electron diffraction. The concept of such computer simulations is described in detail in Refs. [6,7]. Due to quantum-mechanical diffraction patterns, the angular dependence of the electron emission yield shows a rich fine structure, which requires us to use a fine mesh of small angular steps. We therefore consider a range of $\pm 3^{\circ}$ around the $\langle 100\rangle,\langle 110\rangle$ and $\langle 111\rangle$ directions in steps of $\Delta x=\Delta y=0.05^{\circ}$, resulting in characteristic two-dimensional patterns of electron emission probability, $\chi_{\text {theo }}(\theta, \phi)$, where $\theta$ and $\phi$ denote polar and azimuth angles from the axis. The patterns for different electron energies are summed according to the conversion electron branching ratios [16], and smoothed using a Gaussian of $\sigma=0.06^{\circ}$ to account for that part of the experimental angular resolution which is due to the $1 \mathrm{~mm}$ beam spot on the sample. The size and shape of the detector pads is taken into account during fitting by averaging over the simulated yield falling within the angular range $\left(0.26^{\circ} \times 0.26^{\circ}\right)$ of one pad. We fit theoretical emission patterns to the experimental yields $\chi_{\text {ex }}$ according to 
$\chi_{\text {ex }}(\theta, \phi)=S\left[f_{1} \chi_{\text {theo }, 1}(\theta, \phi)+f_{2} \chi_{\text {theo }, 2}(\theta, \phi)+1-f_{1}-f_{2}\right]$,

where $S$ is a scaling factor common to all angles in one pattern, and $f_{1}$ and $f_{2}$ denote the fractions of emitter atoms on two different lattice sites. The random fraction, $f_{\mathrm{R}}=1-\left(f_{1}+f_{2}\right)$, accounts for emitter atoms which cause negligible anisotropies in emission yield, i.e. which are located on sites of very low crystal symmetry or in heavily damaged or amorphous surroundings. Up to six fitting parameters, $S, f_{1}, f_{2}, x_{0}, y_{0}$, and $\phi_{0}$, are simultaneously optimized using non-linear least square fitting routines. Note that the parameter $S$ allows us to define the normalization of the experimental spectra, while $x_{0}, y_{0}$ and $\phi_{0}$ merely describe translational and azimuthal orientation with respect to the detector.

We usually consider substitutional (S), tetrahedral interstitial (T), hexagonal $(\mathrm{H})$, bond center $(\mathrm{BC})$, anti bonding $(\mathrm{AB})$, split $<100\rangle$ (SP) and the socalled $\mathrm{Y}$ and $\mathrm{C}$ sites, as well as <111> and $<100>$ displacements between these sites (cf. Ref. [9] for site descriptions). Best fit results, however, were obtained [Figs. 2 d), e) and f)] for 57(5)\% Tm atoms on lattice sites which are displaced by $d=0.42(8) \AA$ from the $\mathrm{T}$ site, and the rest on random sites. Note that in the case of such small displacements the analysis of the channeling effect can only give values for the mean displacement of the emitter atoms. Consequently, the chi square of the fit showed comparable minima for Tm on sites

Fig. 2. (a), (b) and (c) Channeling patterns from ${ }^{169} \mathrm{Tm} *(0.66 \mu \mathrm{s})$ following room temperature implantation of ${ }^{169} \mathrm{Yb}(32 \mathrm{~d})$ into $n$-Si CZ and annealing to $600^{\circ} \mathrm{C}$ for $10 \mathrm{~min}$. (d), (e) and (f) Best fits of simulated patterns to the experimental yields, corresponding to $56 \%, 58 \%$ and $56 \%$ of emitter atoms on sites which are displaced by $0.42 \AA$ from the $\mathrm{T}$ site.

with static displacements from either $\mathrm{T}$ to $\mathrm{H}, \mathrm{T}$ to $\mathrm{AB}$ or $\mathrm{T}$ to $\mathrm{Y}$ sites. This 
also means that it is not possible to determine whether this is due to static displacements of one specific value along all equivalent crystal axes, or an ensemble of Tm atoms showing several small values of $d$ in the range $\approx 0-0.6 \AA$.

Due to the small recoil of less than $1.1 \mathrm{eV}$ in this nuclear decay, it is very likely that ${ }^{169} \mathrm{Tm}^{*}$ has inherited its lattice site from the mother isotope ${ }^{169} \mathrm{Yb}$. Both $\mathrm{Tm}$ and $\mathrm{Yb}$ are known to occupy interstitial positions in $\mathrm{Si}[2,3,5]$. Remarkably, the interstitial position of $\mathrm{Yb}$ in $\mathrm{Si}$ was first revealed in 1968 by beta emission channeling from the radioactive isotope ${ }^{175} \mathrm{Yb}$ [5]. Later RBS experiments by Andersen et al [2] suggested sites which are displaced from the T site by $0.68 \AA$ along <100> directions, which we have abbreviated above as Y sites. Since their lattice localization was mainly based on qualitative arguments, however, its accuracy should not be overrated, as was already pointed out by Eisen et al [3]. Note that our data would also be compatible with the majority of $\mathrm{Tm}$ on ideal $\mathrm{T}$ sites and some smaller part (10\%) on, e.g., nearby Y sites. Summarizing, we conclude that our emission channeling results are in good agreement with the available RBS data.

While we have chosen a relatively high implantation dose for the long-lived ${ }^{169} \mathrm{Yb}(32 \mathrm{~d})$ in order to limit the detection time, more recent experiments with ${ }^{167} \mathrm{Tm}(9.25 \mathrm{~d}) \rightarrow{ }^{167 \mathrm{~m}} \mathrm{Er}(2.28$ s) were done at considerably reduced doses. Figure $3 \mathrm{a}$ ) shows the $\langle 110\rangle$ channeling pattern from conversion electrons emitted by ${ }^{167 \mathrm{~m}} \mathrm{Er}$ recorded directly following implantation of ${ }^{167} \mathrm{Tm}$ with $5.8 \times 10^{12} \mathrm{~cm}^{-2}$ into $n$-Si:P (3-12 $\Omega \mathrm{cm}, \mathrm{CZ},<111>$ surface). The experimental data could be well fitted by $55 \%$ of Er on near-T sites [Fig. 3b)]. Note that the near-T fraction of Er is probably even higher, due to dechanneling from the damage remaining in this as-implanted sample. We would like to point out that only $4 \%$ of the $4.6 \times 10^{10}$ implanted probe atoms decayed while recording the pattern in Fig. 3a). This clearly shows that, for isotopes with shorter half lives, lattice location studies are feasible for doses around $5 \times 10^{11} \mathrm{~cm}^{-2}$.

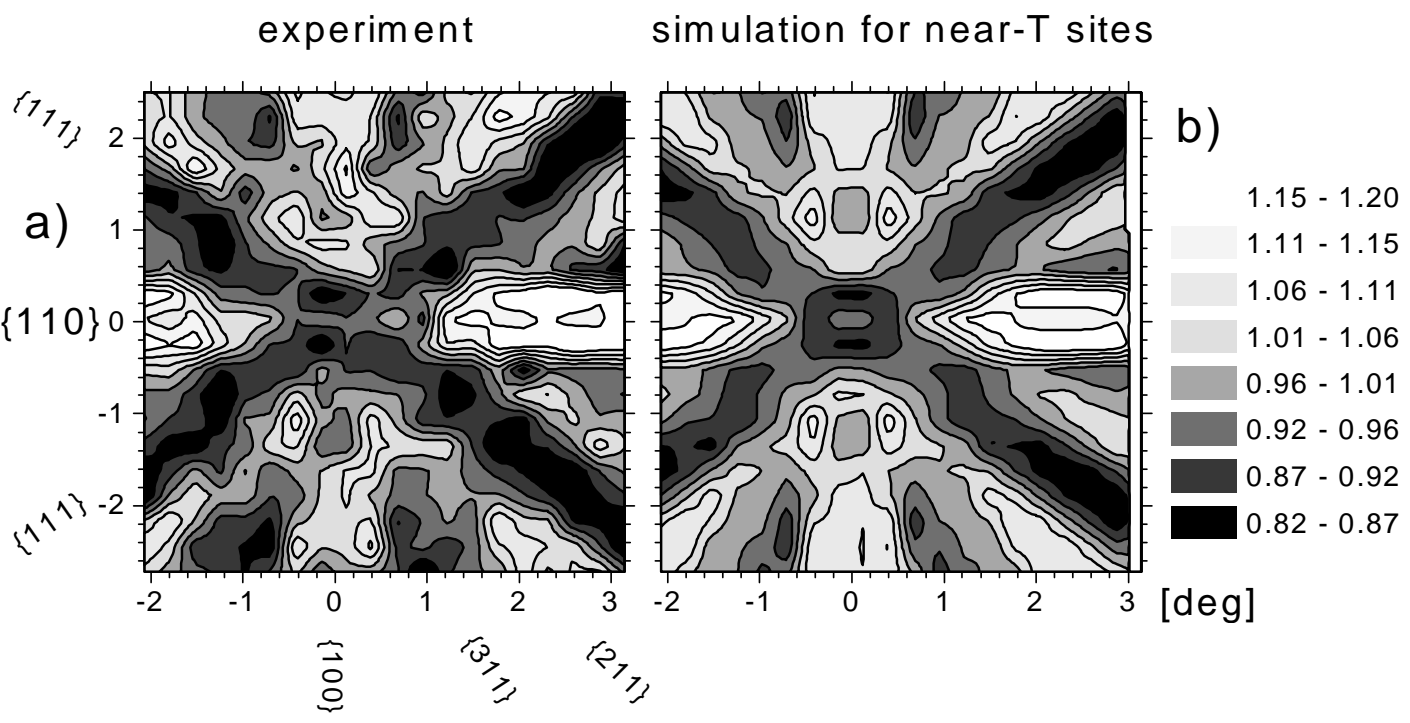

Fig. 3. (a) $\langle 110>$ channeling pattern of the combined intensity of 150,199 and $206 \mathrm{keV}$ conversion electrons from ${ }^{167 \mathrm{~m}} \mathrm{Er}(2.28 \mathrm{~s})$ in $n$-Si CZ. (b) Best fit to the experimental yields, corresponding to $54 \%$ of emitter atoms on sites which are displaced by $0.34 \AA$ from the $\mathrm{T}$ site.

\section{Conclusions}

The use of position-sensitive detectors has further enhanced the capabilities of electron emission channeling as a fast and effective technique to perform quantitative lattice location studies of 
implanted probe atoms. Its strength lies especially in those fields where low solubilities of foreign atoms make accurate ion beam studies difficult or even impossible. In the case of semiconductors, typical examples are the rare earths and transition or noble metals. All of these impurities are of considerable technical interest, while in many cases their lattice sites are not known.

\section{Acknowledgments}

We would like to thank P. Weilhammer, A. Czermak, S.G. Jahn, P. Jalocha, A. Rudge and F. Schopper for providing the position-sensitive detectors, and H. Hofsäss for the permission to use his electron channeling simulation code "Manybeam". J.G.M. and J.G.C. acknowledge JNICT (Portugal) for grants under the Praxis XXI program, A.V. the post-doctoral research program of the Fund for Scientific Research, Flanders (FWO).

\section{References}

[1] L.C. Feldman, J.W. Mayer and S.T. Picraux, Materials Analysis by Ion Channeling (Academic Press, New York, 1982).

[2] J.U. Andersen, O. Andreasen, J.A. Davies and E. Uggerhøj, Radiat. Effects 7 (1971) 25.

[3] F. H. Eisen and E. Uggerhøj, Radiat. Effects 12 (1972) 233.

[4] B. Domeij and K. Bjørkqvist, Phys. Lett. 14 (1965) 127.

[5] E. Uggerhøj and J. U. Andersen, Can. J. Phys. 46 (1968) 543.

[6] H. Hofsäss and G. Lindner, Phys. Rep. 201 (1991) 123.

[7] H. Hofsäss, Hyperfine Interactions 97 (1996) 247.

[8] E. Lægsgaard, Nucl. Instr. Meth. 162 (1979) 93.

[9] U. Wahl, Phys. Rep. 280 (1997) 145.

[10] M. Lindroos and Ö. Skeppstedt, Nucl. Instr. Meth. A 306 (1991) 225.

[11] J. Kemmer and G. Lutz, Nucl. Instr. Meth. A 253 (1987) 365.

[12] U. Wahl, J.G. Correia, A. Czermak, J. De Wachter, W. Dulinski, P. Jalocha, G. Langouche, J.G. Marques, R. Moons, E. Nygård, A. Rudge, F. Schopper, A. Vantomme and P. Weilhammer, to be published.

[13] P. Weilhammer, E. Nygård, W. Dulinski, A. Czermak, F. Djama, S. Gadomski, S. Roe, A. Rudge, F. Schopper and J. Strobel, Nucl. Instr. Meth. A 383 (1996) 89.

[14] S. S. Iyer and Y. H. Xie, Science 260 (1993) 40.

[15] E. Kugler, D. Fiander, B. Jonson, H. Haas, A. Przewloka, H.L. Ravn, D.J. Simon, K. Zimmer and the ISOLDE collaboration, Nucl. Instr. Meth. B 70 (1992) 41.

[16] E. Browne and R.B. Firestone, Table of Radioactive Isotopes (Wiley, New York, 1986). 